\title{
The Effects of Task Load and Change Elements on Change Blindness
}

\author{
Amanda Volkamer ${ }^{1}$ and Kathy Sexton-Radek ${ }^{1}$ \\ ${ }^{1}$ Elmhurst College, Elmhurst, Illinois, USA
}

DOI: https://doi.org/10.47611/jsr.v6i2.297

\begin{abstract}
$\underline{\text { ABSTRACT }}$
The experiment discussed in this paper looks at effects of high and low task loads on detecting change in three conditions: The Control, Change, and Inverse. We wanted to look at how detection of a change may by impacted by the kind of change occurring and the task load. Our first hypothesis predicts that the high-load task will result in more misidentification of the target element than the low-load task. Our other hypothesis predicts the Inverse condition will result in more correctly identified target elements than the Change condition. Our results did not support our second hypothesis and showed that the Inverse Condition was misidentified more so than the Change condition.
\end{abstract}

\section{Introduction}

Change blindness is the inability to notice a change. This phenomenon is related to attention. However, our attention is not perfect and thus, we cannot notice everything in our environment. There are multiple factors that helps our noticing the difference, or lack thereof. Such conditions can be how many elements that changes, such as the whole environment changes or a single element. Another can be a type of distraction or time apart from two comparisons. And another could be if there is a high or low task load that may interfere with our awareness of the change.

Demacheva, Ladouceur, Steinberg, Pogossova, and Raz (2012) investigates the role of attention when watching a magic trick. The participants took an online survey using LimeSurvey online application tool. This included demographic questions, an audiovisual clip, and fourteen specific questions. They continuously played a 15 -second mute video clip of a magic routine to the participants, in which the performer appears to vanish a pen. The participants were to provide two explanations for the magic trick along with a confidence rating using the 5-point Likert scale at the beginning and end of the survey. The results of the experiment showed that thirty-four percent of the participants accurately identified the key moment of when the pen vanished, however, eleven percent of those who correctly identified the key moment could identify what action the magician used to vanish the pen. The decoupling of the when and where pathways of attention modules may play a role in creating effects that seems magical. This study brings up more possible exploration of attention and belief formation.

Drew, Vô, and Wolfe (2013) conducted a research on inattentional blindness and a task load that is wellpracticed by the participants. They asked 24 radiologists to perform a familiar lung-nodule detection task using CT scans. A picture of a gorilla that was much larger than the size of an average nodule was inserted in the last displays presented. The gorilla fade in and out of visibility over five of the slices presented. Eighty-three percent of the radiologists did not see the gorilla. This study supports that inattentional blindness can occur in even well practiced, familiar tasks. The abnormality that is relatively visible can go unnoticed if the abnormality is unexpected. This study not only investigated inattentional blindness but also the presence of expectation bias and its role in inattentional blindness.

Ramachandran and Rogers-Ramachandran (2005) analyzed the effects of expectation in inattentional blindness. They used the gorilla experiment done by Daniel J. Simons and Christopher F. Chabris as their example when 
talking about inattentional blindness. Ramachandran and Rogers explain that our brain creates a script that we follow and when something does not fit the script, we are more likely than not, miss it. Thus, if there is a slight difference and everything else look very similar, we will think that the two pictures displayed are just identical.

Davis, Loftus, Vanous, and Cucciare (2008) conducted three experiment in which to explore the role of change blindness in eyewitness identifications of innocent bystanders. The participants watched a brief film set in a supermarket. There were three characters in the film, the 'continuous innocent' (CI) who walked down the aisle and walked behind a stack of boxes, the perpetrator who emerged and stole a bottle of liquor in that same aisle, and the 'discontinuous innocent' (DI) who was shown immediately afterward in a different aisle. The results showed that more than half of the participants failed to notice the change between the CI and the perpetrator, and among them, more misidentified the CI than the DI. These findings point out that misidentification is likely when there is the illusion of continuity with the idea of unconscious transference.

A study done by Theeuwes (2004) looked at how easily human observers can detect a nonchanging element in a changing environment. The participants of the study were asked to view multiple pairs of pictures and to find the nonchanging line segment in the second picture. The first picture had 4, 8 , or 12 horizontal and vertical $0.6^{\circ}$ line segments, either vertical or horizontal. The second picture shows all but one line segment that was replaced with new one that are tilted to $22.5^{\circ}$ to either side of the vertical or horizontal plane. Participant correctly identified the nonchanging line segment in the condition where there was no interval between the first and second picture, as the nonchanging element seems to "pop out". The condition with the interval between the displays showed more inefficiency of identifying the line segment than the first condition without the interval, although search for just one nonchanging element rather than multiple was supported to be very efficient regardless of the interval. The results of the study suggest that we are not blind to things that do not change.

Stone (2005) focuses on attention and change blindness. An experiment done by Nilli Lavie of University College London shown that subjects who are shown two different faces with a task in between the two pictures fail to notice the difference. When there is not a task in between the two photos, the subjects noticed that the two photos are different. This is how a magician's trick usually goes unnoticed by the observer.

Our research study is a $3 \times 2$ within study design to test multiple questions; three levels of change and two levels of the task loads. How does the high and low task load impact the identification of the target element? We believe that if the participants are presented with a high-load task, they would misidentify the target element more than if they were presented a low-load task. How does it compare across all conditions? Across all the conditions, we believe that the high-load task would have the same effect across all the conditions in which the participants will misidentify the target element more than the low-load task. However, out of the three conditions, we expect the Change condition to have higher misidentification in the high task load and low task load as compared to the other two conditions. Does one condition result in higher identification of the target element over the over conditions? According to prior studies done, we believe the Inverse condition would have higher identification than the Change condition. We believe the Control condition would have higher correct responses over both other conditions.

\section{Method}

\section{Participants}

There were 10 participants in this study: 8 females and 2 males. The participants were Elmhurst College students in psychology classes and were assigned to the date and time they had signed up for. All the participants were collegeaged; 6 freshmen, 1 sophomore, and 3 juniors. The participants were rewarded with 1 psych credit towards their psychology class for their participation. 


\section{Materials}

We created thirty pairs of pictures that consisted of shapes. There were ten pairs for each condition: the Control- no change, the Change- same surrounding, different object, and the Inverse condition. The Control group picture pair would show no difference of the shapes. The Change condition would show one shape changed in the second picture of the pair. The Inverse condition would show all the shapes from the first picture have swapped places in the second picture. The shapes presented will be either squares, circle, triangle, stars, or hearts. Five of the ten pairs consisted of only five objects as it would the low-load task. The other five of the ten pairs consisted of ten objects as it would the high-load task. We put the thirty pairs on a PowerPoint which the first picture of the pair, the blank slide in between, and the second picture of the pair is timed for 5 seconds each and the slide that stated "Trial \#x" timed for 12 seconds (See Figure 1). The picture pairs were put in a randomized order based on a random number draw.

The participants completed a worksheet, which was graded later based on the answer key we created which has thirty trials, one for each pair presented to them. Each trial had questions that asks whether they can identify the target element and if so, what was the change they saw. Each trial had a 5-point Likert scale confidence rating (1= Not Confident, $5=$ Very Confident) to judge the participant's confidence in each trial. The participants completed a survey that asked questions for demographic information.

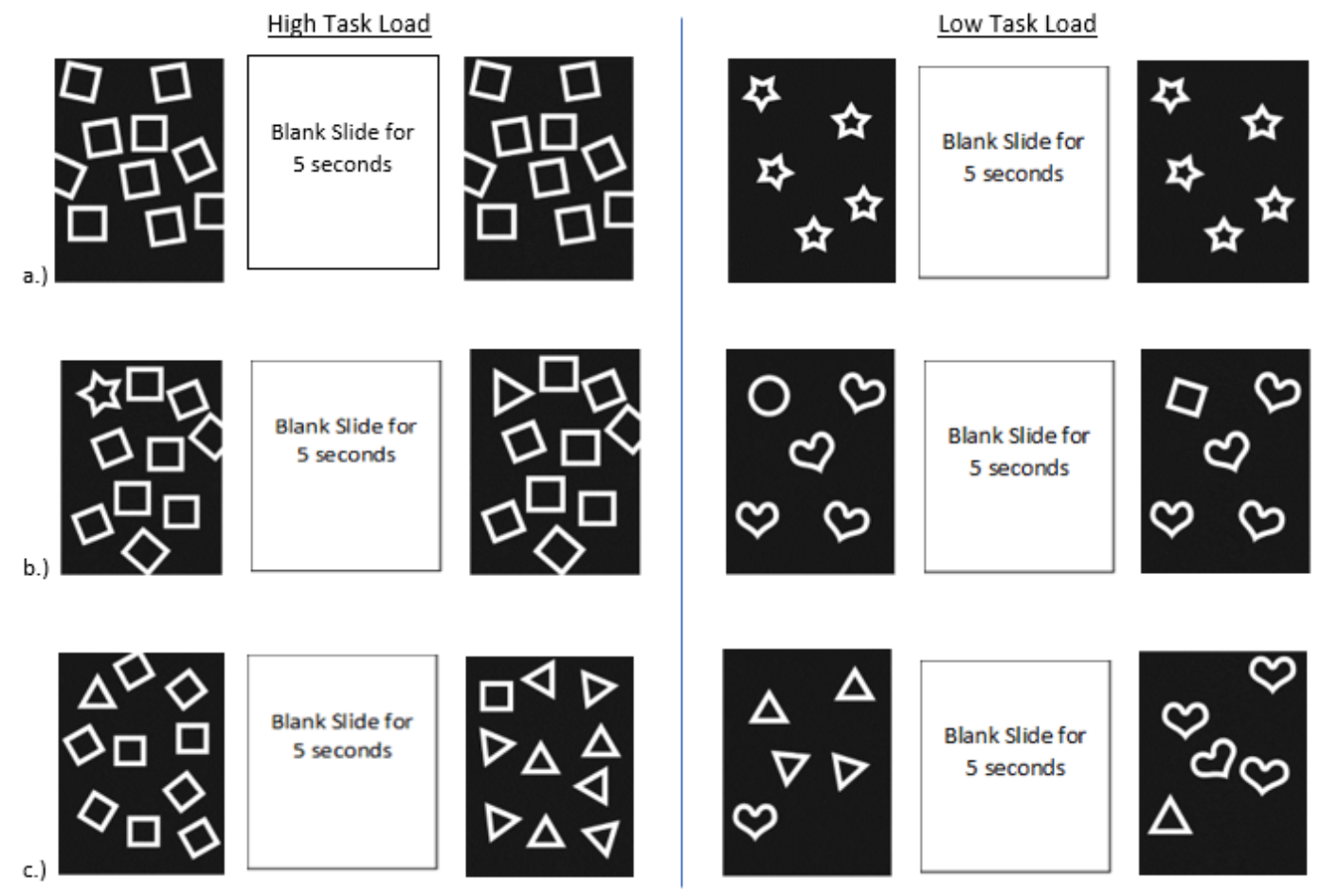

Figure 1. The three conditions within High-Tasks and Low-Tasks. a.) The control condition shows no changes from the first to second picture. b.) The change condition shows one shape is changed from the first picture to the second picture, and c.) shows the inverse condition where the shapes switched from the first picture to the second picture.

\section{Procedure}

Each condition from different times received the exact same procedure as this is a within-subject design. The participants were asked to sign the consent form. Once we received all the consent form, we passed out a worksheet for each participant. One of our experimenters told the participants the instructions. Next, we presented the PowerPoint on the 
projector screen in the classroom. The participants were asked to fill out the worksheet and answer each question for each trial. Once all the trials are presented, the participants turned in their worksheet and filled out the survey. After filling out the survey, the participants received a debriefing paper and the blue psych credit before they left. The overall, each session of the experiment lasted 30 minutes.

\section{Results}

We ran ANOVA tests for our three datasets. Our first dataset corresponds with the first question on the worksheet asking if the participant detected any change. The ANOVA results showed a main effect in Change $(\mathrm{F}(2,18)=4.72$, $\mathrm{p}-.023)$ and no effect in the Load $(\mathrm{F}(1,9)=.141, \mathrm{p}<1)$ and contrary to our predictions, the Change and Load interaction $(\mathrm{F}(1,8)=.935, \mathrm{p}<1)$ (See graphs for the first dataset). We ran descriptive tests on the Change to locate where the effect was occurring. The comparison of the control condition $(\mathrm{M}=4.8, \mathrm{SD}=0.4)$ and the inverse condition $(\mathrm{M}=4.0, \mathrm{SD}=0.87)$ was statistically significant $(\mathrm{p}=.006)$. The comparison of the control condition and the change condition $(\mathrm{M}=4.5, \mathrm{SD}=0.68)$ was not significant $(\mathrm{p}=.193)$, as was the change condition and the inverse condition $(\mathrm{p}=.168)$.

The second dataset corresponds with the second question on the worksheet asking if the participant could correctly identify what specifically changed. The ANOVA showed a main effect in Change $(F(2,18)=7.81, p=$ $.004)$. The Load did not have a main effect $(\mathrm{F}(1,9)=1.39, \mathrm{p}<1)$, as did the Change and Load interaction $(\mathrm{F}(1,18)$ $=2.63, \mathrm{p}<1$ ), contrary to our predictions (See graphs for the second dataset). The follow up descriptive test showed the comparison of the control condition $(\mathrm{M}=4.8, \mathrm{SD}=0.4)$ and the change condition $(\mathrm{M}=3.4, \mathrm{SD}=1.5)$ has a significance $(\mathrm{p}=.004)$. The comparison of the control condition and the inverse condition $(\mathrm{M}=2.9, \mathrm{SD}=1.56)$ is also significant $(p=.002)$. The comparison of the change condition and the inverse condition is not significant $(p=.467)$.

Our third dataset corresponds with the confidence rating based on the 5-point Likert scale for each trial. The ANOVA results showed a main effect in $\operatorname{Load}(\mathrm{F}(1,9)=9.18, \mathrm{p}=.014)$. There was not any effect in Change $(\mathrm{F}(2$, $18)=2.62, \mathrm{p}<1)$ and the Change and Load interaction $(\mathrm{F}(1,18)=.21, \mathrm{p}<1)$ (See graphs for the third dataset). The Low-load Task condition had a higher mean than the High-load Task condition ( $M=4.3, M=4.0)$, though the probability was not given.

\section{Discussion}

Our results from our first dataset which asked the question if a difference between two pictures could be detected showed that there is a significant difference between the Control condition and the Inverse condition rather than the Change condition. Based on the means from each condition, this finding does not support our hypothesis stating that the Change condition, overall, would result in higher misidentification than the other two conditions. The opposite occurred as the Inverse condition appeared to have a significant difference from the control. The Control condition had a significantly higher mean than the Inverse condition.

The results of the second dataset, which asked if the difference could be correctly identified, if there was a difference, showed something quite interesting. Though the results did not show any significance between the Change condition and the Inverse condition, the difference between each condition and the Control condition, is significant. The Control condition had a significantly higher mean than the Change and Inverse conditions. This is to be expected because the Control condition showed no change, and therefore the second question would not apply. There is a greater significant gap between the Control condition mean and the Inverse condition mean than the Control and the Change conditions. This result also does not support our hypothesis and rather supports the opposites, in which there seemed to have been a higher misidentification of the difference in the Inverse condition.

One of the limiting factors of our study is our small sample size. We had only 10 participants who showed up for our study. The results could be due to this small sample size and the significance we found in our results could 
very well disappear in a larger sample size due to a generalization effect. This is something to consider for any further research or replications of our study to see if our results would similarly apply to a larger population.

Another area of concern with our study was the issue of time. The PowerPoint we presented to the participants was on a set time loop. In our first group, there was a failure to explain that the PowerPoint is timed, therefore there is limited time to answer the questions on the worksheet. The second group, however, did receive the word of caution about the limit on time. This may have an impact on the results as there were some questions that were not answered and were marked to not receive any points. This can skew the data numbers and is a confounding variable that is possible. This can also apply more specifically for the third dataset that is concerned with the confidence rating as there were some participants who did not fill out the confidence rate scale.

The importance that our study raise to awareness is the fact that our detection of change could be in part due to the type of change that occurs. This draws upon our attention to our environment. We may notice one thing that changes and not that the entire environment changed. The task load in our study was to see if there was the effect of low or high load task in our detection of change and our attention to the details. This area of study is beneficial in testimonies in criminal trials and police work.

\section{References}

Davis, D., Loftus, E. F., Vanous, S., \& Cucciare, M. (2008). 'Unconscious transference' can be an instance of ‘change blindness'. Applied Cognitive Psychology, 22(5), 605-623. Doi: 10.1002/acp.1396.

Demacheva, I., Ladouceur, M., Steinberg, E., Pogossova, G., \& Raz, A. (2012). The applied cognitive psychology of attention: A step closer to understanding magic tricks. Applied Cognitive Psychology, 26(4), 541-549. Doi: $10.1002 /$ acp. 2825

Drew, T., Vô, M. H., \& Wolfe, J. M. (2013). The invisible gorilla strikes again: Sustained inattentional blindness in expert observers. Psychological Science, 24(9), 1848-1853.

Ramachandran, V. S., \& Rogers-Ramachandran, D. (2005). How Blind Are We?. Scientific American Mind, 16(2), 96-95.

Stone, A. (2005). Focus Pocus. Discover, 26(12), 10.

Theeuwes, J. (2004). No Blindness for Things That Do Not Change. Psychological Science, 15(1), 65-70. Doi:10.1111/j.0963-7214.2004.01501011.x 